\title{
Correlação entre a espessura corneana central e o comprimento axial ocular nos portadores de glaucoma e em olhos normais
}

\author{
Correlation between central corneal thickness and axial length in patients \\ with glaucoma and normal eyes
}

\author{
Paula Resende Aquino de Assis Pereira Mello ${ }^{1}$ \\ Sérģio Henrique Sampaio Meirelles ${ }^{2}$ \\ Haroldo Vieira de Moraes Júnior ${ }^{3}$
}

Trabalho realizado no Departamento de Oftalmologia do Hospital Universitário Clementino Fraga Filho da Universidade Federal do Rio de Janeiro (UFRJ) - Rio de Janeiro (RJ) - Brasil.

${ }^{1}$ Pós-graduanda nível mestrado do Serviço de Oftalmologia do Hospital Universitário Clementino Fraga Filho, Universidade Federal do Rio de Janeiro (UFRJ) Rio de Janeiro (RJ) - Brasil.

${ }^{2}$ Professor Assistente da Universidade Gama Filho e Chefe do Serviço de Oftalmologia do Hospital Municipal da Piedade. Doutor em Oftalmologia pela UFRJ Rio de Janeiro (RJ) - Brasil.

${ }^{3}$ Professor Adjunto e Coordenador do Curso de Pósgraduação de Oftalmologia da Faculdade de Medicina da UFRJ - Rio de Janeiro (RJ) - Brasil.

Endereço para correspondência: Paula Resende Aquino de Assis Pereira Mello. Rua Desembargador Canedo, 375 - Apto. 502 - Muriaé (MG)

CEP 36880-000

E-mail: paula.raap@gmail.com

Recebido para publicação em 25.04.2008

Última versão recebida em 07.06.2009

Aprovação em 15.06.2009

\begin{tabular}{|c|}
\hline RESUMO \\
\hline $\begin{array}{l}\text { Objetivo: Correlacionar a espessura corneana central com o comprimento } \\
\text { axial ocular nos portadores de glaucoma primário de ângulo aberto, com } \\
\text { glaucoma primário de fechamento angular e indivíduos com olhos normais. } \\
\text { Métodos: A amostra foi constituída de } 94 \text { olhos de } 94 \text { pacientes, divididos } \\
\text { em três grupos compostos por } 33 \text { olhos de } 33 \text { pacientes portadores de } \\
\text { glaucoma primário de ângulo aberto, } 30 \text { olhos de } 30 \text { pacientes com glaucoma } \\
\text { primário de fechamento angular e } 31 \text { olhos normais de } 31 \text { indivíduos. A } \\
\text { espessura corneana e o comprimento axial do olho foram obtidos pela } \\
\text { paquimetria ultrassônica e ecobiometria, respectivamente. Resultados: A } \\
\text { média da espessura corneana central foi de } 535,1 \mu \text { mo glaucoma primário } \\
\text { de fechamento angular; } 520,6 \mu \text { mo glaucoma primário de ângulo aberto } \\
\text { e } 519,2 \mu m \text { nos olhos normais (p=0,18). A média do comprimento axial do } \\
\text { globo ocular nos portadores de glaucoma primário de fechamento angular } \\
\text { foi de } 22,16 \text { mm e nos grupos com glaucoma primário de ângulo aberto e } \\
\text { olhos normais foram de } 22,68 \text { mm e } 22,64 \text { mm, respectivamente (p=0,13). } \\
\text { Não houve correlação significativa entre a espessura corneana central e } \\
\text { comprimento axial do globo ocular nos grupos com glaucoma primário } \\
\text { de fechamento angular (r=-0,085; } p=0,65 \text { ), glaucoma primário de ângulo } \\
\text { aberto(r=-0,070; } \mathrm{p}=0,69 \text { ) eolhos normais ( } \mathrm{r}=-0,120 ; \mathrm{p}=0,52 \text { ). Conclusão: Os } \\
\text { resultados deste trabalho sugerem não haver correlação entre a espessura } \\
\text { corneana central e o comprimento axial do globo ocular nos portadores de } \\
\text { glaucoma e indivíduos com olhos normais. }\end{array}$ \\
\hline
\end{tabular}

Descritores: Córnea/anatomia \& histologia; Glaucoma de ângulo fechado; Glaucoma de ângulo aberto; Campos visuais

\section{INTRODUÇÃO}

O glaucoma é uma doença multifatorial com grande variedade de apresentações clínicas e achados histopatológicos ${ }^{(1)}$. É caracterizado por neuropatia óptica com alterações típicas da cabeça do nervo óptico, como perda do tecido neural e aumento da escavação do disco óptico ${ }^{(2)}$, e defeitos característicos do campo visual, causada por morte das células ganglionares, o que acarreta perdas funcionais irreversíveis ${ }^{(3)}$.

A paquimetria corneana deve ser considerada em todos portadores ou suspeitos de glaucoma, no diagnóstico e na avaliação da eficácia do tratamento do glaucoma ${ }^{(4-5)}$.

A ecobiometria no glaucoma de ângulo estreito é de grande importância, tanto para o diagnóstico como para a evolução clínica e também na avaliação 
pré-operatória destes olhos, no sentido de nos orientar a respeito do risco de eventual ocorrência de complicações cirúrgicas que estes olhos comumente apresentam ${ }^{(6)}$.

Alguns autores avaliaram a relação entre a espessura corneana e o comprimento axial do olho em 1.084 olhos glaucomatosos. Concluíram, que não houve significância estatística na associação entre a espessura corneana e o comprimento axial do olho, sugerindo serem ocorrências independentes ${ }^{(7)}$.

Numerosos estudos têm demonstrado correlação entre a espessura corneana central e a pressão intraocular ${ }^{(4-5,8-10)}$ e outros relacionados com dimensões oculares (comprimento axial, profundidade da câmara anterior) e progressão do glaucoma ${ }^{(2,11-16)}$. Porém, existem poucos relatos na literatura ${ }^{(7,17-19)}$ associando a espessura corneana e o comprimento axial do globo ocular nos portadores de glaucoma, o que nos motivou a realizar este trabalho.

O objetivo deste estudo é verificar a correlação entre a espessura corneana central e o comprimento axial dos olhos com glaucoma primário de ângulo aberto, com os de glaucoma de fechamento angular e olhos normais.

\section{MÉTODOS}

A amostra foi constituída de 94 olhos de 94 pacientes, sendo dividida em três grupos: glaucoma primário de ângulo aberto, glaucoma primário de fechamento angular e indivíduos com olhos normais, os quais foram avaliados no serviço de oftalmologia do Hospital Clementino Fraga Filho da Universidade Federal do Rio de Janeiro, no período de dezembro de 2006 a julho de 2007. Os pacientes foram submetidos a exame de acuidade visual, refração, tonometria de aplanação, gonioscopia, biomicroscopia do nervo óptico, perimetria computadorizada, ecobiometria e paquimetria ultrassônica.

Para análises estatísticas, somente um olho de cada paciente foi incluído no estudo, de modo randomizado, exceto nos pacientes que só apresentavam um olho que obedecia aos critérios de inclusão.

Os critérios de inclusão foram: idade maior que 35 anos, ausência de uso prévio de colírios mióticos ou midriáticos, história negativa de cirurgia intraocular ou procedimentos a laser, ausência de catarata e qualquer doença intraocular, que pudesse interferir com os resultados campimétricos e fundoscópicos de glaucoma. Os pacientes com glaucoma primário de ângulo aberto e de fechamento angular apresentaram pelo menos duas medidas da pressão intraocular maior ou igual a $22 \mathrm{mmHg}$, em ocasiões diferentes. Os indivíduos normais do grupo controle apresentavam exame oftalmológico dentro da normalidade com pressão intraocular menor que $21 \mathrm{mmHg}$. Quanto ao exame gonioscópico, os pacientes com glaucoma primário de ângulo aberto e os de olhos normais, apresentavam ângulo aberto sem anormalidades e os pacientes com glaucoma de fechamento angular, apresentavam ângulo estreito (classificação de Shaffer grau 0,1 ou 2) e presença, ou não, de sinéquia anterior periférica. Todos apresentavam alterações característi- cas de glaucoma ao exame do nervo óptico e do campo visual (exceto o grupo com olhos normais).

Consideramos como alterações características de glaucoma no nervo óptico, assimetria da relação escavação/disco óptico maior que 0,2 entre os olhos, afinamento do anel neurorretiniano com diâmetros da rima neural menor ou igual a 0,1 , aumento da escavação $(>0,6)$, "notching", hemorragias do disco óptico ${ }^{(20)}$.

Como critério de defeito de campo visual glaucomatoso, adotamos o seguinte: presença de escotomas paracentrais ou arqueados, degrau nasal maior que 10 graus de extensão e aumento dos índices do campo visual na perimetria computadorizada. Ainda em relação ao campo visual, adotamos: defeito de pelo menos 3 pontos adjacentes não periféricos, diminuição de sensibilidade de $5 \mathrm{~dB}$, com um dos pontos apresentando baixa sensibilidade de pelo menos $10 \mathrm{~dB}^{(3)}$. O defeito campimétrico deve corresponder à lesão do nervo óptico. Todos os pacientes realizaram pelo menos dois exames de campo visual confiáveis e reprodutíveis, usando o programa 24-2, estratégia Full-threshold do aparelho Humphrey, com estímulo branco em fundo branco.

Foram excluídos do estudo os pacientes que apresentavam outros tipos de glaucoma, hipertensão ocular sem diagnóstico confirmado de glaucoma, diabetes mellitus com glicemia acima de 120 mg/dL ou com retinopatia diabética e hipertensão arterial sem tratamento.

O exame gonioscópico foi realizado com auxílio de lentes com espelhos (lente de Goldman), que são colocadas em contato com a córnea, intermediadas por substância viscoelástica (ex. metilcelulose $2 \%$ ). Os olhos foram anestesiados com colírio de cloridrato de proparacaína a $0,5 \%$. O exame foi realizado sob visibilização através de microscópio (lâmpada de fenda) sempre pelo mesmo examinador e em sala iluminada, entre $13 \mathrm{~h}$ e $30 \mathrm{~min}$ e 15 h e $30 \mathrm{~min}$.

Para a comparação da amplitude de diferentes ângulos da câmara anterior do olho, utilizamos a classificação de Shaffer, que avalia em graus numéricos a amplitude do ângulo de acordo com a descrição anatômica ${ }^{(1,21-22)}$ : ângulo aberto (grau 4) amplitude do ângulo 35-45 - impossível fechamento; ângulo aberto (grau 3) - amplitude do ângulo 20-35 - impossível fechamento; ângulo moderadamente estreito (grau 2) - amplitude $20^{\circ}$ - possível fechamento; ângulo extremamente estreito (grau 1) - amplitude $10^{\circ}$ - provável fechamento; ângulo completamente ou parcialmente fechado (grau 0) - amplitude $0^{\circ}$ - fechamento presente ou iminente.

As medidas paquimétricas foram realizadas utilizando o princípio da ultrassonografia de modo $\mathrm{A}$, com o paquímetro DGH technology, INC 5100 (EUA). O exame procedeu-se com os pacientes deitados em posição supina, com o olhar direcionado para o teto. Os olhos foram anestesiados com colírio de cloridrato de proparacaína a $0,5 \%$, dois minutos antes do exame. O aparelho foi ajustado para a velocidade de $1.636 \mathrm{~m} / \mathrm{seg}$ e uma precisão de 5 micra. A sonda foi colocada perpendicularmente ao ápice da córnea.

As medidas biométricas foram efetuadas pelo método de ultrassonografia de contato (Accutome/Accusonic). O exame foi 
realizado com os pacientes sentados e o médico posicionado lateralmente ao doente. Após a instilação de colírio anestésico (cloridrato de proparacaína a $0,5 \%$ ), a sonda A foi encostada axialmente à superfície central da córnea, ou seja, perpendicular, tocando-a de modo delicado, evitando identação. Durante o exame, o paciente manteve-se em posição primária do olhar e o examinador indicou um ponto de fixação para o olho contralateral.

Os exames de paquimetria e ecobiometria foram realizados sempre pelo mesmo examinador, realizando todas as medidas entre $13 \mathrm{~h} 30 \mathrm{~min}$ e $15 \mathrm{~h} 30 \mathrm{~min}$.

Em cada olho foi considerada a média de cinco medidas confiáveis da espessura corneana e do comprimento axial do globo ocular.

Todos os indivíduos com olhos normais e os portadores de glaucoma foram orientados quanto aos objetivos e a metodologia empregada, assinando, após esta orientação, o termo de consentimento livre e esclarecido. O protocolo da pesquisa foi aprovado pelo comitê de ética do Hospital Universitário Clementino Fraga Filho, onde foi realizado o trabalho.

Foram utilizados os sequintes testes estatísticos: Análise de Variância “one-way", para comparações de variáveis contínuas (numéricas) entre os três grupos (glaucoma primário de ângulo aberto, glaucoma primário de fechamento angular e controle), o teste de comparações múltiplas de Tukey foi aplicado para identificar quais os grupos que diferem entre si, o coeficiente de correlação de Pearson (r) para medir o grau de associação linear entre a espessura corneana e o comprimento axial do olho.

Foi considerado significativo valor de $\mathrm{p}$ menor que 0,05 . A análise estatística foi processada pelo "software" R para Windows, versão 2.4.0, ano 2006.

\section{RESULTADOS}

O grupo 1 foi constituído de 33 olhos de 33 pacientes com glaucoma primário de ângulo aberto (GPAA), sendo 20 do sexo feminino, 13 do sexo masculino, 13 brancos, 10 pardos, 10 negros, com idade média de 66,21 anos, variando de 45 a 83 anos.

O grupo 2 foi composto por 30 olhos de 30 pacientes portadores de glaucoma primário de fechamento angular (GPFA), sendo 22 do sexo feminino, 8 do sexo masculino, 13 brancos, 7 pardos, 10 negros, com idade média de 67,13 anos, variando de 47 a 79 anos.

O grupo 3 foi formado de 31 olhos de 31 indivíduos normais (ON), sendo 27 do sexo feminino, 4 do sexo masculino, 13 brancos, 5 pardos e 13 negros, com idade média de 64,14 anos, variando de 50 a 78 anos.

Observou-se que não existe associação significativa $(\mathrm{p}=0,056)$ entre o sexo e o grupo. Existe uma forte tendência de o grupo ON apresentar proporção de mulheres (87\%) maior que o grupo GPAA $(60 \%)$. Teste estatístico: qui-quadrado $\left(\chi^{2}\right)$. (Tabela 1$)$

Pelo teste estatístico de análise de variância (ANOVA), observou-se que não existe diferença significativa na idade $(p=0,47)$ entre os grupos. (Tabela 2)
A tabela 3 mostra a média, desvio padrão, mediana, mínimo e máximo da espessura corneana central em cada grupo, sendo a média de $535,1 \mu \mathrm{m}$ nos pacientes com glaucoma primário de fechamento angular, $520,6 \mu \mathrm{m}$ no grupo com glaucoma primário de ângulo aberto e 519,2 $\mu \mathrm{m}$ no grupo com olhos normais, mostrando que não houve diferença significativa $(\mathrm{p}=0,18)$ pelo teste de análise de variância (ANOVA) - fator único.

Não houve diferença significativa entre a média, desvio padrão, mediana, mínimo e máximo do comprimento axial do globo ocular nos três grupos, sendo a média de $22,16 \mathrm{~mm}$ no grupo com GPFA, 22,68 mm no grupo com GPAA, 22,64 mm no grupo de $\mathrm{ON}$, como está demonstrado na tabela $3(\mathrm{p}=0,13)$.

A tabela 4 fornece a média, desvio padrão, mediana, mínimo e o máximo do comprimento axial ocular e da espessura corneana central segundo a classificação de Shaffer referente ao aspecto gonioscópico. Os graus 0 e 1 foram agrupados na classe 1, o grau 2 foi agrupado na classe 2, o grau 3 foi agrupado na classe 3 e o grau 4 agrupado na classe 4 . Os graus 0 e 1 foram agrupados na mesma classe, devido ao pequeno número de pacientes com o grau $0(n=3)$. Observou-se que existe diferença significativa no comprimento axial $(\mathrm{p}=0,011)$ entre as classes. Pelo teste de comparações múltiplas de Tukey, identificou-se apenas que a classe 4 foi significativamente maior que a classe 1. Não existe diferença significativa na espessura corneana entre as classes $(p=0,25)$.

A análise comparativa entre os grupos (Gráfico 1) segundo o coeficiente de correlação de Pearson mostrou que não existe correlação significativa entre a espessura corneana central e o comprimento axial do globo ocular no grupo com GPFA $(\mathrm{r}=-0,085 ; \mathrm{p}=0,65 ; \mathrm{n}=30)$, no grupo com GPAA $(\mathrm{r}=-0,070$; $\mathrm{p}=0,69 ; \mathrm{n}=33)$ e no grupo de $\mathrm{ON}(\mathrm{r}=-0,120 ; \mathrm{p}=0,52 ; \mathrm{n}=31)$.

\section{DISCUSS $\tilde{A} O$}

Alguns trabalhos relatam a não relação entre o comprimento axial do olho e a espessura corneana central, revelando que são independentes e que córneas delgadas não estão associadas com olhos longos ${ }^{(7,17-19)}$. No entanto, nenhum destes estudos comparou grupos de glaucoma primário de ângulo aberto, com grupos de glaucoma primário de fechamento angular e indivíduos com olhos normais.

Tomamos a precaução de não incluir no trabalho, pacientes glaucomatosos que fizeram ou faziam uso de colírio miótico ou que foram submetidos à iridectomia cirúrgica ou a laser. Isto porque, de acordo com alguns autores, as drogas mióticas

\begin{tabular}{|c|c|c|c|c|c|c|}
\hline \multirow{2}{*}{$\begin{array}{l}\text { Grupo/ } \\
\text { sexo }\end{array}$} & \multicolumn{2}{|c|}{ GPFA } & \multicolumn{2}{|c|}{ GPAA } & \multicolumn{2}{|c|}{ ON } \\
\hline & $n$ & $\%$ & $\mathbf{n}$ & $\%$ & $\mathbf{n}$ & $\%$ \\
\hline Masculino & 8 & 26,67 & 13 & 39,39 & 4 & 12,90 \\
\hline Feminino & 22 & 73,33 & 20 & 60,61 & 27 & 87,10 \\
\hline
\end{tabular}




\begin{tabular}{|lcccccccc|}
\hline \multicolumn{7}{c}{ Tabela 2. Média, desvio padrão, mediana, mínimo e máximo da idade dos três grupos } \\
Variável & Grupo & $\mathbf{n}$ & Média & Desvio padrão & Mediana & Mínimo & Máximo & p-valor (ANOVA) \\
Idade (anos) & GPFA & 30 & 67,13 & 8,99 & 70,00 & 47,00 & 79,00 \\
& GPAA & 33 & 66,21 & 10,44 & 65,00 & 45,00 & 83,00 \\
\multicolumn{7}{ll}{ GPFA= glaucoma primário de fechamento angular; GPAA= glaucoma primário de ângulo aberto; ON= olhos normais } & 78,00 \\
\hline
\end{tabular}

\begin{tabular}{|c|c|c|c|c|c|c|c|c|}
\hline Variável & Grupo & $\mathbf{n}$ & Média & Desvio padrão & Mediana & Mínimo & Máximo & p-valor (ANOVA) \\
\hline \multirow[t]{3}{*}{ Comprimento axial (mm) } & GPFA & 30 & 22,16 & 1,11 & 22,06 & 20,17 & 24,76 & \multirow{3}{*}{0,13} \\
\hline & GPAA & 33 & 22,68 & 1,07 & 22,44 & 21,13 & 26,54 & \\
\hline & ON & 31 & 22,64 & 1,10 & 22,56 & 20,86 & 26,62 & \\
\hline \multirow[t]{3}{*}{ Espessura corneana $(\mu \mathrm{m})$} & GPFA & 30 & 535,01 & 44,66 & 530,00 & 439,00 & 651,00 & \multirow{3}{*}{0,18} \\
\hline & GPAA & 33 & 520,06 & 31,27 & 523,00 & 441,00 & 583,00 & \\
\hline & ON & 31 & 519,02 & 34,85 & 524,00 & 420,00 & 575,00 & \\
\hline
\end{tabular}

\begin{tabular}{|c|c|c|c|c|c|c|c|c|}
\hline Variável & Classificação & $\mathbf{n}$ & Média & Desvio padrão & Mediana & Mínimo & Máximo & p-valor (ANOVA) \\
\hline \multirow[t]{4}{*}{ Comprimento axial (mm) } & Classe 1 & 19 & 22,14 & 1,11 & 22,06 & 20,17 & 24,23 & \multirow{4}{*}{0,011} \\
\hline & Classe 2 & 11 & 22,20 & 1,17 & 22,21 & 20,60 & 24,66 & \\
\hline & Classe 3 & 48 & 22,66 & 1,04 & 22,48 & 21,13 & 26,54 & \\
\hline & Classe 4 & 16 & 22,66 & 1,22 & 22,54 & 20,86 & 26,62 & \\
\hline \multirow[t]{4}{*}{ Espessura corneana $(\mu \mathrm{m})$} & Classe 1 & 19 & 541,90 & 42,30 & 531,00 & 503,00 & 651,00 & \multirow{4}{*}{0,250} \\
\hline & Classe 2 & 11 & 523,40 & 48,20 & 511,00 & 439,00 & 598,00 & \\
\hline & Classe 3 & 48 & 522,30 & 34,37 & 529,00 & 420,00 & 583,00 & \\
\hline & Classe 4 & 16 & 512,80 & 27,24 & 515,00 & 465,00 & 564,00 & \\
\hline
\end{tabular}

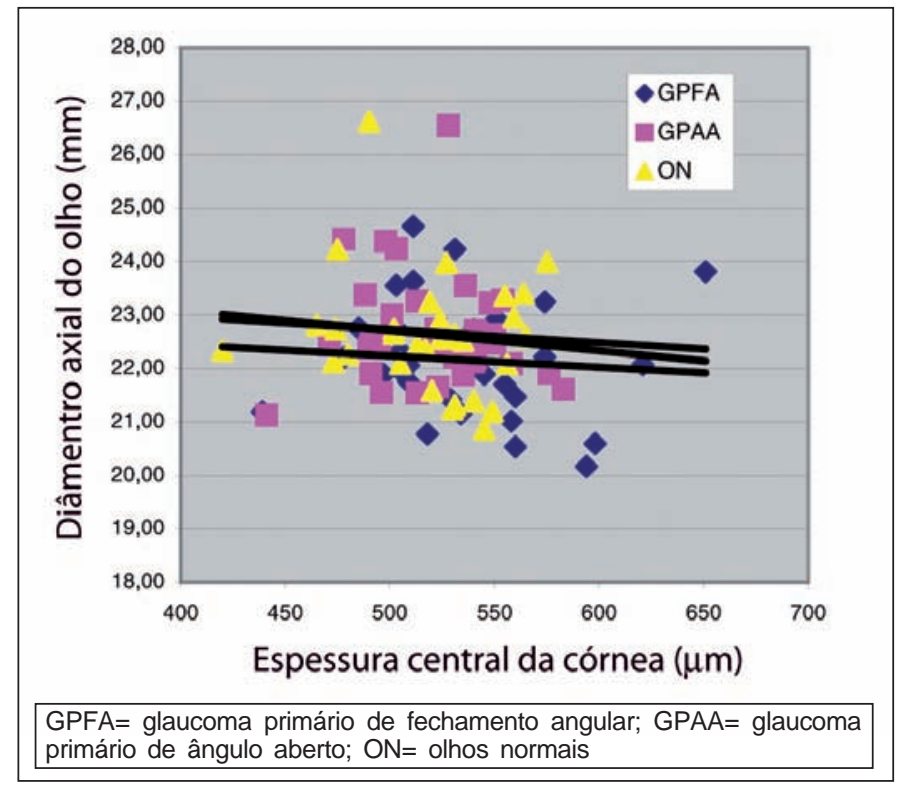

Gráfico 1 - Correlação entre o diâmetro axial do olho e a espessura central da córnea nos três grupos induzem um espasmo acomodativo, causando espessamento e posição anteriorizada do cristalino, reduzindo a profundidade axial da câmara anterior e estreitando o seio camerular ${ }^{(23-24)}$.

Nos grupos com glaucoma primário de ângulo aberto e glaucoma primário de fechamento angular, a maioria dos pacientes usava de um a três colírios hipotensores oculares, no momento da paquimetria. No entanto, de acordo com a literatura pesquisada, não consideramos que este fato possa ter influenciado no resultado final ${ }^{(23,25-26)}$.

A tabela 3 mostra que não houve diferença significativa na espessura corneana central entre os grupos com glaucoma primário de ângulo aberto, glaucoma primário de fechamento angular e olhos normais, embora a espessura corneana fosse ligeiramente maior no grupo com glaucoma de fechamento angular.

Esses resultados corroboram com a maioria dos estudos publicados $^{(11-12,18)}$. Um estudo publicado em 2001, os autores relataram que a espessura central da córnea foi menor em pacientes com glaucoma de pressão normal $(538 \mu \mathrm{m})$ e maior em pacientes hipertensos oculares $(570 \mu \mathrm{m})$, sendo similar em glaucoma primário de ângulo aberto e pessoas saudáveis (547 e 
$554 \mu \mathrm{m}$, respectivamente) $)^{(18)}$. Resultados semelhantes foram encontrados em outro trabalho com 22 pacientes com glaucoma de pressão normal ( $\mathrm{EC}=521 \mu \mathrm{m}), 49$ com glaucoma primário de ângulo aberto $(\mathrm{EC}=543 \mu \mathrm{m}), 44$ hipertensos oculares $(\mathrm{EC}=583 \mu \mathrm{m})$ e 18 pessoas do grupo controle $(E C=552 \mu \mathrm{m})^{(12)}$.

Não vimos referência ao horário em que foi realizada a paquimetria na maioria dos trabalhos pesquisados. De acordo com estudos de alguns autores, não houve significância estatística na variação da espessura central da córnea durante o dia, portanto, somente uma medida da espessura corneana é suficiente ${ }^{(8)}$. No entanto, outro estudo relatou que a espessura corneana é variável durante o período de 24 horas $^{(27)}$.

Em relação aos estudos sobre paquímetros publicados na literatura, os autores relataram que ambos os paquímetros, óptico e ultrassônico, são de mesma acurácia, sendo que o paquímetro ultrassônico apresenta maior variabilidade inter e intra-observador do que o método óptico. Ambos registram medidas de espessura corneana muito comparáveis, apesar do método ultrassônico produzir estimativa de espessura corneana ligeiramente mais alta $^{(9,13-14)}$. O nosso estudo e a maioria dos trabalhos citados anteriormente utilizaram o paquímetro ultrassônico para medida da espessura corneana central.

A comparação estatística dos valores do comprimento axial do globo ocular nos três grupos demonstrou que não existe diferença significativa entre os grupos, como pode ser observado na tabela 3. Em relação aos resultados, observamos que o grupo com glaucoma primário de fechamento angular, teve o comprimento axial ligeiramente menor em comparação aos outros dois, cujos valores foram similares. No entanto, essa diferença não foi estatisticamente significativa.

Estes achados não estão de acordo com os trabalhos por nós compulsados $^{(15-16,28-29)}$. Um estudo relatou diferenças estatisticamente significativas no comprimento axial entre os grupos com glaucoma crônico simples $(\mathrm{OD}=30,08 \mathrm{~mm} / \mathrm{OE}=30,20 \mathrm{~mm})$ e com glaucoma agudo primário $(\mathrm{OD}=28,08 \mathrm{~mm} / \mathrm{OE}=28,90 \mathrm{~mm})$, com valores médios maiores nos olhos portadores de glaucoma crônico simples ${ }^{(13)}$. Outro estudo também revelou que o grupo de pacientes com glaucoma crônico de ângulo estreito e o grupo de pacientes com glaucoma agudo apresentaram diâmetro axial total dos olhos significativamente menores que os olhos normais, ou quando comparados com glaucoma de ângulo aberto. Os resultados foram, para glaucoma crônico de ângulo estreito $(21,7 \mathrm{~mm})$, glaucoma agudo $(21,8 \mathrm{~mm})$, olhos normais (23,0 mm), glaucoma de ângulo aberto $(23,8 \mathrm{~mm})$. Os autores realizaram biometria dos olhos contralaterais somente nos casos de glaucoma agudo e maligno quando estes ocorreram em um olho único ${ }^{(15)}$.

Porém, outros autores não encontraram diferença significativa no comprimento axial de 30 pacientes portadores de glaucoma primário de ângulo aberto e 30 pessoas com olhos normais $^{(28)}$.

No nosso estudo, observou-se que não houve correlação significativa entre a espessura corneana central e o comprimento axial dos olhos com glaucoma primário de ângulo aberto, com glaucoma de fechamento angular e de olhos normais (gráfico 1).

Nossos resultados estão de acordo com outros trabalhos tanto nos casos de glaucoma como nos indivíduos normais $^{(7,10,17,19)}$. Um estudo publicado em 2005 com 546 pacientes glaucomatosos (1.084 olhos) constatou que não houve significância estatística na associação entre o comprimento axial do olho e a espessura central corneana. A média da espessura corneana, para todos os olhos foi de 543,1 $\mu \mathrm{m}$ (variação de 367,0 a 754,0 $\mu \mathrm{m}$ ) e a média do comprimento axial foi 23,44 (variação de 17,05 a 34,64 mm) ${ }^{(7)}$. Em outro trabalho com resultados semelhantes ao anterior, com o total da amostra de 140 olhos de 140 pacientes glaucomatosos, houve correlação significativamente positiva entre a espessura central da córnea e a espessura escleral, mas não entre o erro refrativo ou o comprimento axial do olho. A média do comprimento axial foi 23,70 (variação de 15,50 a $30,55 \mathrm{~mm}$ ) e da espessura corneana foi 547,3 (variação de 452,0 a 728,0 $\mu \mathrm{m})^{(19)}$.

Alguns autores relataram que a espessura corneana central parece ser uma variável independente e que não se relaciona significativamente com outras dimensões oculares ${ }^{(10)}$.

Analisamos estatisticamente o comprimento axial e a espessura corneana em relação à classificação de Shaffer na amostra geral (94 pacientes), que foram divididos em classes 1 , 2, 3 e 4, referente ao aspecto gonioscópico.

Na tabela 4, podemos observar que existe diferença significativa no comprimento axial entre as classes $(1,2,3,4)$. Pelo teste de comparações múltiplas de Tukey, somente a classe 4 teve o comprimento axial maior que o da classe 1. Este fato pode ser explicado pelo valor do comprimento axial de 22,66 mm ser uma média amostral, não é fixo e, portanto, tem um desvio padrão. O desvio padrão da classe 4 é maior que a da classe 3, portanto os dados da classe 4 tem uma "variação maior". Este resultado já era esperado por nós.

Em relação à espessura corneana central, nenhuma diferença significativa foi encontrada entre as classes $(1,2,3,4)$, segundo a classificação de Shaffer, referente ao aspecto goniocópico (Tabela 4).

Nosso estudo limitou-se apenas correlacionar a espessura corneana central com o comprimento axial dos olhos nos portadores com glaucoma primário de ângulo aberto, glaucoma primário de fechamento angular e indivíduos com olhos normais. Portanto, não relacionamos com outros fatores, como: pressão intraocular, erro refrativo, gravidade ou progressão do glaucoma. Seriam necessárias mais pesquisas para melhor avaliar a associação entre estas variáveis (comprimento axial e espessura corneana central) nos portadores de glaucoma e indivíduos com olhos normais.

\section{CONCLUSÃO}

Os resultados deste estudo sugerem não haver correlação entre a espessura corneana central e o comprimento axial do globo ocular nos olhos dos pacientes portadores de glaucoma e indivíduos com olhos normais. 


\section{ABSTRACT}

Purpose: To evaluate and to correlate the central corneal thickness with the ocular axial length in patients with primary open-angle glaucoma, primary angle-closure glaucoma and individuals with normal eyes. Methods: The sample was composed of 94 patients' eyes, divided into three groups constituted of 33 eyes of 33 primary open-angle glaucoma patients, 30 eyes of 30 primary angle-closure glaucoma patients and 31 normal eyes of 31 individuals. The central corneal thickness and the axial length were measured by ultrasonic pachymeter and biometry by A-scan ultrasound, respectively. Results: The results showed a mean of $535.1 \mu \mathrm{m}$ central corneal thickness in primary angle-closure glaucoma group, $520.6 \mu \mathrm{m}$ in primary open-angle glaucoma group and $519.2 \mu \mathrm{m}$ in normal eyes $(\mathrm{p}=0.18)$. The ocular axial length on primary angle-closure glaucoma group was $22.16 \mathrm{~mm}$ and on primary open-angle glaucoma and normal eyes group was $22.68 \mathrm{~mm}$ and $22.64 \mathrm{~mm}$, respectively $(\mathrm{p}=0.13)$. There was no significant correlation between corneal thickness and axial length in the primary angle-closure glaucoma group $(r=-0.085 ; \mathrm{p}=0.65)$, the openangle glaucoma group $(\mathrm{r}=-0.070 ; \mathrm{p}=0.69)$ and the normal eyes group ( $r=-0.120 ; p=0.52)$. Conclusions: The results of this assay suggest that there is no correlation between the central corneal thickness and the axial length in patients with glaucoma and individuals with normal eyes.

Keywords: Cornea/anatomy \& histology; Glaucoma, angle-closure; Glaucoma, open-angle; Visual fields

\section{REFERÊNCIAS}

1. Allingham RR, Damji KF, Freedman S, Sayoto EM, Shafronov G, Shields M B. Shields' textbook of glaucoma. $5^{\mathrm{a}}$ ed. Philadelphia: Lippincott Williams \& Wilkins; 2005. p.724.

2. Hewitt AW, Cooper RL. Relationship between corneal thickness and optic disc damage in glaucoma. Clin Experiment Ophthalmol. 2005;33(2):158-63. Comment in: Clin Experiment Ophthalmol. 2006;34(1):93; author reply 93-4.

3. Costa VP, Carvalho CA. Perimetria computadorizada: um guia básico de interpretação. Rio de Janeiro: RioMed livros; 1995. p.133.

4. Sakata K, Figueira ALM, Guimarães ACP, Schimtt AJ, Scapucin L, Barros LGR, et al. Estudo da correlação entre pressão intraocular e espessura corneana central (projeto glaucoma). Arq Bras Oftalmol. 2000;63(5):355-8.

5. Lee GA, Khaw PT, Ficker LA, Shah P. The corneal thickness and intraocular pressure story: where are we now? Clin Experiment Ophthalmol. 2002;30(5): 334-7.

6. Betinjane AJ. Ecobiometria na patologia ocular, no glaucoma e nos tumores intraoculares. In: Abreu G. Ultrassonografia ocular: atlas e texto. $3^{\underline{a}}$ ed. Rio de Janeiro: Cultura Médica; 2002. p.43-50.

7. Shimmyo M, Orloff PN. Corneal thickness and axial length. Am J Ophthalmol. 2005;139(3):553-4
8. Cronemberger S, Calixto N, Costa LT, Soares FM. Corneal thickness and daily curve of intraocular pressure in suspected and glaucomatous patients. Arq Bras Oftalmol. 2005;68(2):185-8.

9. Kohlhaas M, Boehm AG, Spoerl E, Pursten AD, Grein HJ, Pillunat LE. Effect of central corneal thickness, corneal curvature, and axial length on applanation tonometry. Arch Ophthalmol. 2006;124(4):471-6.

10. Whitacre MM, Stein RA, Hassanein K. The effect of corneal thickness on applanation tonometry. Am J Ophthalmol. 1993;115(5):592-6.

11. Lowe RF. Aetiology of the anatomical basis for primary angle-closure glaucoma: Biometrical comparisons between normal eyes and eyes with primary angleclosure glaucoma. Br J Ophthalmol. 1970;54(3):161-9.

12. Copt RP, Thomas R, Mermoud A. Corneal thickness in ocular hypertension, primary open-angle glaucoma, and normal tension glaucoma. Arch Ophthalmol. 1999;117(1):14-6. Comment in: Arch Ophthalmol. 1999;117(1):104-5.

13. Calixto N, Cronemberger S. Glaucoma simples x glaucoma agudo. Estudo ecobiométrico. Arq Bras Oftalmol. 1986;49(1):1-8.

14. Kim JW, Chen PP. Central corneal pachymetry and visual field progression in patients with open-angle glaucoma. Ophthalmology. 2004;111(11):2126-32.

15. Susanna Junior R, Nicolela MT, Fukushima JT. Comparação biométrica entre diferentes tipos de glaucoma e sua importância clínica. Arq Bras Oftalmol 1988;51(3):116-7.

16. Chen YF, Wang TH, Hung PT. Influence of axial length on visual field defects in primary open-angle glaucoma. J Formos Med Assoc. 1997;96(12):968-71.

17. Ehlers N, Hansen FK, Aasved H. Biometric correlations of corneal thickness Acta Ophthalmol (Copenh). 1975;53(4):652-9.

18. Singh RP, Goldberg I, Graham SL, Sharma A, Mohsin M. Central corneal thickness, tonometry, and ocular dimensions in glaucoma and ocular hypertension. J Glaucoma. 2001;10(3):206-10. Comment in: J Glaucoma. 2002;11(4) 371-2; author reply 372; J Glaucoma. 2002;11(6):543.

19. Oliveira C, Tello C, Liebmann J, Ritch R. Central corneal thickness is not related to anterior scleral thickness or axial length. J Glaucoma. 2006;15(3):190-4.

20. Jonas JB, Bergua A, Schmitz-Valckenberg P, Papastathopoulos KI, Budde WM Ranking of optic disc variables for detection of glaucomatous optic nerve damage. Invest Ophthalmol Vis Sci. 2000;41(7):1764-73.

21. Chan RY, Smith JA, Richardson KT. Anterior segment configuration correlated with Shaffer's grading of anterior chamber angle. Arch Ophthalmol. 1981 99(1):104-7.

22. Khatana AK, Spaeth GL. Glaucoma de ângulo fechado. In: Vander JF, Gault JA Segredos em Oftalmologia: respostas necessárias ao dia-a-dia, em rounds, na clínica, em exames orais e escritos. Porto Alegre: Artmed; 2001. p.152-66.

23. Poinoosawmy DS, Nagasubramanian S, Brown NP. Effect of pilocarpine on visual acuity and on the dimensions of the cornea and anterior chamber. Br J Ophthalmol. 1976;60(10):676-9.

24. Kobayashi H, Kobayashi K, Kiryu J, Kondo T. Ultrasound biomicroscopic analysis of the effect of pilocarpine on the anterior chamber angle. Graefe's Arch Clin Exp Ophthalmol. 1997;235(7):425-30.

25. Kaminski S, Hommer A, Koyuncu D, Biowski R, Barisani T, Baumgartner I Influence of dorzolamide on corneal thickness, endothelial cell count and corneal sensibility. Acta Ophthalmol Scand. 1998;76(1):78-9.

26. Lass JH, Eriksson GL, Osterling L, Simpson CV; Latanoprost Corneal Effects Study Group. Comparison of the corneal effects of latanoprost, fixed combination latanoprost-timolol, and timolol. A double-masked, randomized, one-year study Ophthalmology. 2001;108(2):264-71. Comment in: Ophthalmology. 2001 108(12):2152.

27. Harper CL, Boulton ME, Bennett D, Marcyniuk B, Jarvis-Evans JH, Tullo AB et al. Diurnal variations in human corneal thickness. Br J Ophthalmol. 1996; 80(12):1068-72. Erratum in: Br J Ophthalmol. 1997;81(2):175.

28. Sihota R, Dada T, Gupta R, Lakshminarayan P, Pandey R. Ultrasound biomicroscopy in the subtypes of primary angle closure glaucoma. J Glaucoma. 2005 14(5):387-91.

29. Chisholm IA, Drance SM, Chauhan BC. The glaucoma suspect: differentiation of the future glaucomatous eye from the non-glaucomatous suspect eye. I. Ultrasonic measurements and eye-wall stress. Graefe's Arch Clin Exp Ophthalmol. $1989 ; 227(1): 17-20$. 